\title{
A!
}

This is an electronic reprint of the original article.

This reprint may differ from the original in pagination and typographic detail.

Nieminen, Mika; Kirjonen, Markus

\section{Keeping It Real!}

Published in:

Augmented Reality, Virtual Reality, and Computer Graphics

DOI:

10.1007/978-3-030-58465-8_4

Published: $31 / 08 / 2020$

Document Version

Peer reviewed version

Please cite the original version:

Nieminen, M., \& Kirjonen, M. (2020). Keeping It Real! Investigating Presence in Asymmetric Virtual Reality. In L.

T. De Paolis, \& P. Bourdot (Eds.), Augmented Reality, Virtual Reality, and Computer Graphics (pp. 50-60).

(Lecture Notes in Computer Science; Vol. 12242). SPRINGER. https://doi.org/10.1007/978-3-030-58465-8_4

This material is protected by copyright and other intellectual property rights, and duplication or sale of all or part of any of the repository collections is not permitted, except that material may be duplicated by you for your research use or educational purposes in electronic or print form. You must obtain permission for any other use. Electronic or print copies may not be offered, whether for sale or otherwise to anyone who is not an authorised user. 


\title{
Keeping It Real! \\ Investigating Presence in Asymmetric Virtual Reality
}

\author{
Mika P. Nieminen \\ Aalto University School of Science, P.O Box 15400 FI-00076 Aalto, Finland \\ mika.nieminen@aalto.fi \\ Markus Kirjonen \\ Aalto University School of Business, P.O Box 15400 FI-00076 Aalto, Finland \\ markus.kirjoneneaalto.fi
}

\begin{abstract}
In this paper we discuss the necessity to preserve the sense of presence in virtual reality (VR). A high sense of presence has proven advantages but is also very fragile to interruptions. We outline scenarios where interaction and communication between persons inside and outside virtual environments are necessary and assess challenges for maintaining the immersed user's sense of presence in such cases. We also use existing literature to outline an experiment that allows us to try out different methods of collaboration between immersed users and external facilitators in order to discern their effect on presence.
\end{abstract}

Keywords: Virtual reality, sense of presence, immersion, collaborative problemsolving, asymmetric virtual reality.

\section{Introduction}

This paper discussed the concept of presence, or the feeling of being naturally part of an immersive virtual environment (VE). Our goal is to better understand the many uses and challenges of presence. Our goal is to increase the effectiveness of VR in some key tasks in collaborative problem-solving and co-design and to find suitable communication and interaction practices when working in asymmetric VR environments.

Presence, the user's subjective sense of "being there," is a key element of virtual reality [1-3]. It has been shown that when a user experiences a high sense of presence, they respond to the virtual world as they would to the real one, displaying realistic responses such as emotions, reactions, and behaviors $[4,5]$.

This ability to elicit realistic responses makes VR a promising medium for studying human behavior, and as such, it has seen adoption in the fields of user research, participatory design, co-design, psychology, psychiatry, and more. In many cases, the ability to simulate scenarios and environments at the click of a mouse is a significant advantage over more commonplace methods of immersing users in a desired context. Rather than trying to establish a sense of "being there" through design artifacts and narratives, or

adfa, p. 1, 2011.

(C) Springer-Verlag Berlin Heidelberg 2011 
having the user physically travel to the environment in question, researchers can anchor them in a context that would otherwise be impossible, impractical, or cost-prohibitive to set up. As long as the user maintains a high sense of presence, they will respond to the virtual environment as if they were actually there in real life.

Unfortunately, presence in virtual reality is not guaranteed [6,7]. A user's sense of presence can fluctuate, or even break completely [8], in response to events in both the virtual and real world. One of the most common reasons for this sort of break in presence is interference from the external world; i.e. hearing somebody talking or having them try to get your attention $[8,9]$. This is problematic, as interaction between a subject and facilitator is a staple of many design and research approaches. At any point during the experiment, the non-immersed facilitator may want to ask the immersed user to describe their experience, point to something in the virtual environment, or provide guidance if they are unable to proceed with the task at hand. All of these interactions carry with them a significant possibility of shattering the immersed user's sense of presence if not properly integrated into the virtual environment.

This paper makes the case for the search of context-appropriate methods of interaction and communication between immersed users and external facilitators that are conducive to maintaining the immersed user's sense of presence, thus preserving the virtual environment's ability to elicit realistic behaviors and emotions.

\section{The Meaning of Being There}

The lately booming interest in VR technology is shared among different variants of augmented reality (AR), mixed reality (MR) or virtual reality. We will concentrate on the right edge of the Milgram and Kishino's [10] virtuality continuum, namely fully immersive virtual environments and augmented virtuality (see Fig. 1).

While we are eagerly waiting for the fully immersive photorealistic massively multiuser environments like the Oasis depicted in the 2011 bestseller Ready Player One [11], we must conclude that the most effective and meaningful VR collaboration requires active participation from parties external to the VR environment. The question of how to facilitate seamless interaction, collaboration and communications between a fully immersed user in the VR and the accompanying users in the real life remains. This kind of setting where users have access to variable levels of immersion is commonly referred to as asymmetric virtual reality. Some examples of interactions designed to support such asymmetry in collocated contexts are ShareVR [12] and RoleVR [13]. Peter et al.

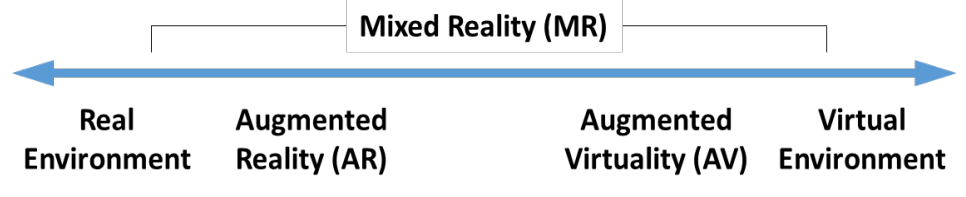

Virtuality Continuum (VC)

Fig. 1. Virtuality continuum [10]. 
[14] identified a rich set of conceptual features designed to support interactions between immersed users and external users in such a context (which they dubbed "VR-Guides"). These features were further categorized into four categories:

- View-related features, allowing the VR Guide to observe the virtual scene from multiple perspectives,

- Manipulation features, allowing the VR Guide to control, add, and delete objects in the scene

- Meta-part features, allowing the VR Guide to control the simulation, e.g. start and end it, trigger a virtual event

- Monitoring features, allowing the VR Guide to visualize parts of VE that the user has explored or is paying attention to, gather affective computing data (e.g. heart rate or skin conductivity), statistics about the VE experience such as time lapsed.

Presence is often described as the user's subjective psychological response to a virtual environment and the experience of "being there" [1-3]. Presence is needed for eliciting realistic behavior i.e. reactions and emotions in a virtual environment $[4,5]$, and it has also been shown that the higher the user's sense of presence is, the more realistic their responses are [15].

\section{Application Areas that Require Presence}

Our hypothesis throughout this paper is that maintaining high level of presence enables seamless collaboration and leads to good flow on interactive activities. VR opens new possibilities for user-centered design, user experience (UX) design, participatory design and co-design as long as the necessary interactions between all stakeholders can be kept as natural as possible. Brade et al. [16] concluded that virtual environments can substitute real environments for user experience studies if they achieve high presence. The use of VR environments and tools can enable cost effective prototyping and evaluation of products, services, and environments as shown by Rebelo et al. [17]. Some suitable contexts for VR enabled usability and UX research and co-design are hospitals and ATMs [18] and architectural design [19].

Another field that benefits from high level of presence is exposure therapy. This behavioral therapy technique involves exposing a patient to the source of their phobia, without danger, in an effort to gradually reduce the anxiety they associate with it. As the range of a patient's possible phobias is endlessly broad, one can easily imagine why virtual reality would be useful in this endeavor. With the click of their mouse, a therapist can simulate any stimuli, ranging from spiders to large crowds (given a VE built for such simulations). Indeed, as Koller et al. [20] stated, virtual reality exposure therapy (VRET) is potentially a major improvement over both using real-world stimuli (invivo exposure therapy), which may be difficult or cost-prohibitive to procure, and relying on the patient's imagination (in-situ exposure therapy). Of course, VRET requires the virtual environment to elicit realistic reactions and emotions on the part of the patient, and thus, presence has been heavily researched in this context [20-23]. 
More broadly, virtual reality has seen utilization in psychology for similar reasons; as Diemer et al. [5] describe, it allows us to study human behavior in highly controlled and customizable settings. Examples here include Yee \& Bailenson [24] using virtual reality avatars to study the effect of self-presentation on behavior, Peck et al. [25] studying the effect of embodying an avatar representing a minority race in reducing racial bias, and Slater et a. [26] repeating the infamous Milgram obedience experiment in virtual reality, none of which would be possible without the medium. As before, the usefulness of these experiments depends on the virtual environment's ability to elicit realistic reactions.

\section{Presence: On/Off}

Presence in VR is not guaranteed. The level of presence that a user experiences depends on factors relating to hardware, software, and the individual user in question, as discussed below. Nor is it static. The level of presence that a user feels can fluctuate in response to events in both the virtual and real world, and can even break entirely, as discussed by Slater \& Steed [8]. This is undesirable, as it reduces the virtual environment's ability to elicit realistic responses.

\subsection{Things making Presence}

Presence can be increased by various factors. Some of these relate to the virtual reality hardware itself. Cummings \& Bailenson [7] highlight the tracking level, field of view, update rate, and stereoscopy as having a particularly significant effect on the user's sense of presence. These features are important as they allow for a low level of latency between the user's sensory data and sense of self movement, as emphasized by Slater [27]. In other words, it is important for what the user sees to accurately match how they feel their body moving, a dynamic which is heavily dependent on the quality of technology being used. Less impactful features include image quality and resolution [7]. Software-related design choices within the virtual environment are also highly relevant in establishing a high sense of presence. For instance, having the environment appropriately react to the user's actions is important. This includes realistic shadows and lighting [6], having objects that represent living being behaving appropriately in response to the user [27], and the user's ability to participate in and modify the virtual environment [28]. Finally, as presence is a subjective phenomenon, the user in question has a significant effect on the level of presence that is provided by the virtual environment. Samana et al. [29] found that individual traits on the part of the user that affect their level of experienced presence include imagination, empathy, level of anxiety, cognitive style, dissociative tendencies, and more. The user's situational interest is also an important factor in how present the user feels [5,30], along with their general state of mind and recent history at the time of the experience. [6]. 


\subsection{Things breaking Presence}

Just as some factors can increase a user's sense of presence, others can decrease or even break a user's sense of presence completely. Slater \& Steed [8] categorized reasons for breaks in presence into two main categories: external causes (sensory information from the external, real world intruding upon or contradicting what is happening in the virtual world) and internal causes (something is "wrong" in the virtual world, such as improper physics, incorrect or missing sounds, or objects not acting realistically). They also identified other categories, such as experiment-related causes, personal causes, attention-related causes, and spontaneous causes for breaks in presence. In their ranking, external causes such as hearing people talking in the background, or experiencing external touches or forces, were found to be two of the most common reasons for breaks in presence. This seems to be the case for any situation where the user interacts with, or becomes aware of, the external world [9,31]. This susceptibility to external interruptions presents a problem in virtual reality; while a user is immersed, external people in the real world may want to interact or communicate with them going as far as nudging them to get their attention before talking [9]. Problems relating to the virtual reality hardware being used have also been shown to be potential causes for breaks in presence. Two common factors here include awareness of the virtual reality equipment and control apparatus [32]. In addition, cybersickness, which can be caused by technology-related factors such as latency in tracking or low refresh rates, has been well-established as a significant source of breaks in presence [33].

\section{$5 \quad$ Measuring Presence}

Presence can be conceptually split to two categories: physical and social. IJsselsteijn et al. [34] declare that "The physical category refers to the sense of being physically located somewhere, whereas the social category refers to the feeling of being together (and communicating) with someone." Measuring presence has two main approaches. In subjective measures participants provide a conscious assessment of their psychological state in relation to the mediated environment, while objective measures try to capture person's responses automatically and without conscious deliberation [35]. Van Baren and IJsselsteijn [36] offer a complete compendium of measurements. They list subjective measures which include various questionnaires, continuous assessment, qualitative and psychophysical measures, while objective measures include psychophysical measures, neural correlates and behavioral and task performance measures. While the objective measures sound tempting given the limitations of subjective approaches [33], most researchers have concluded that for now, objective measures are a good addition to asking users to describe their subjective experience, not a replacement $[7,37,38]$.

While questionnaires are the most common form of measuring presence, there has been some discussion on their limitations. As Slater [39] points out, by asking about one's sense of presence, you are bringing into question the phenomenon that you're supposed to be measuring, which may affect the phenomenon itself. As questionnaires are usually administered afterwards, they rely on the user's memory of presence, rather 
than the experience of presence itself [40, 41]. This also means that you must break presence in order to leave the virtual environment and fill out a questionnaire [42]. To circumvent this one can of course implement self-reporting during the experiment, rather than afterwards. One option if to implement questionnaires into the virtual reality environment as explored by Schwind et al. [41, 43].

\section{Discussion}

Based on the state of the art and our research requirements we were able to outline an experiment consisting of an asymmetric VR environment suited for creative problem solving that is discussed here in further detail. The current design will only fully support the VR experience, while the asymmetric desktop part is used by the experiment facilitator to enforce seamless integration of the various interactivity features in a Wizard of Oz fashion (for examples see [44-46])

\subsection{VR environment}

Our VR test environment is built using the Unity game engine along with Valve Corporation's SteamVR plugin to power our integration with the headset and controllers. Our interactivity features require local networking and VOIP communication, for which we planning to use pre-existing frameworks such as Mirror ${ }^{1}$, an open source networking framework for Unity, and WebRTC Video Chat ${ }^{2}$, a Unity VOIP plugin with virtual reality support. The hardware used is an off-the-shelf HTC VIVE Pro 2.0 Starter Kit equipped with the VIVE Wireless adapter to enable untethered movement.

Our virtual environment is split into two builds, each featuring a different set of functionality; one is for the immersed user and will run on the VR headset, while the other is for the external user and will run on a nearby desktop PC. The VR build is primarily focused on first-person interactions with the environment to perform the required tasks, while the desktop build is designed for guidance and communication-related features. The non-VR build shows a birds-eye view of the virtual environment and the view of the immersed user. These builds are synchronized in real-time via a local area network. In addition to these two Unity builds, we have also developed a web-based dashboard for preparing upcoming sessions and going through previous session data.

\subsection{The Experiment}

The experiment design was guided by our requirements to enable seamless yet asymmetric interaction between the fully immersed VR user and their counterpart in the real world. We wanted to support collaborative creative problem solving and be able to test several different kinds of presence preserving interactions.

\footnotetext{
https://github.com/vis2k/Mirror

2 http://because-why-not.com
} 
The whole of the experiment is expected to last no more than an hour including all transitions and putting on and taking off the VR equipment. Upon their departure each participant is given two movie tickets (value 20€) to thank them for their valuable contribution. Selection criteria for the test subjects include equal gender balance, 18-45 years old, no earlier observed or diagnosed VR or motion sickness, and that they do not professionally work with VR technologies.

Chronologically the experiment test setup consists of four parts with expected durations in parenthesis.

Welcoming the participants, pre-task questionnaire and informed consent (10 min)

The test participants are guided into our VR lab, and they fill a short pre-test questionnaire to verify the selection criteria and to collect their informed consent to take part to the test. Each user is informed of the common ethical consideration about the experiment [47]: we are testing the VR application and not the users, emphasize that the software is not ready and may include errors, tell the users they may stop the test at any time, explain that activities both the VR environment and the lab room are recorded for further analysis, but the results are anonymized and their identities are kept confidential.

\section{Introduction to VR gear and establishing presence baseline (10 $\mathrm{min})$}

In the start of the experiment, the user can try the VR headset and controllers in one of the three different example VR environments, chosen to represent low, medium and high physical presence with a between-subjects design. This will allow the users to learn the basics of selecting and activating objects inside VR environments. Filling a presence questionnaire after this initial trial takes place after removing the headset and before the introduction of our VR environment.

\section{Asymmetric creative problem-solving task (15min)}

We chose to use a virtual escape room as the template for our experiment. Real life escape rooms have become excessively popular lately, so we hope the goals and common practices in them are familiar to our test subjects. This lowers the need to draw detailed instructions for the tasks, which would inevitably hinder the immersion to the actual task.

During the creative problem-solving task, the immersed VR user works through a series of activities with variable difficulty in a single room environment. Some tasks are designed to specifically require external assistance and guidance from the non-immersed party/experiment facilitator. After each task, we will measure the immersed user's sense of presence while they are in the virtual environment using integrated VR presence questionnaires (as seen in [42]). By integrating the questionnaires into virtual reality, we are able to reduce the downtime between each task and avoid having to reorientate the user to the environment. After filling out the questionnaire, the user is able to move onto the next task, thus starting a new iteration of this task-questionnaire cycle. In addition to the presence questionnaires at the end of each task, the user can also self- 
report their sense of presence at any point throughout the experiment using a built-in mechanism, a handheld slider, similar to [8].

During the experiment the user must collaborate with the external party to complete some of the tasks. Together with trying out several interaction methods accompanied with an in-VR presence measurement, we can assess the effects of interactions between the virtual and real world on the experienced presence of the immersed user. Among the escape room tasks each immersed user will be reached through two different type of interaction conduits. Some of the conduits are considered highly disruptive to the presence such as shouting instructions to the immersed user while they complete the task. A practice common to current VR use, when a single person is playing a game and others offer their advice in a loud voice to make the VR user to hear them. More subtle conduits aim to enable communication and interactivity in a context-appropriate way and aim to preserve the immersed user's sense of presence by interacting through objects in the VR environment. Example of such presence-preserving methods include talking to the immersed user via an available telephone or walkie-talkie (handheld twoway radio transceiver), or pointing at objects in a way adherent to the laws of that specific virtual environment; for instance, in the case of a fantasy themed setting a sparkling fairy pointing at objects may be appropriate, but it would make less sense in a more realistic environment. Possible interaction methods, with examples of varying levels of integration into the virtual environment, are explored in Table 1.

Table 1. Communication and interaction methods for asymmetric VR environments.

\begin{tabular}{|l|l|}
\hline $\begin{array}{l}\text { Verbal communication } \\
\text { The immersed user and exter- } \\
\text { nal facilitator interact directly } \\
\text { through speech. The effect on } \\
\text { presence is presumed to } \\
\text { depend on the level of } \\
\text { embodiment that is applied to } \\
\text { the external user's voice. }\end{array}$ & $\begin{array}{l}\text { - } \begin{array}{l}\text { Unintegrated voice (the facilitator's voice comes from } \\
\text { the real, external world, with no modifications, e.g. per- } \\
\text { son shouting next to you) }\end{array} \\
\text { Disembodied voice (the facilitator's voice is heard in } \\
\text { the virtual reality, with no integration to the VE) } \\
\text { Object-embodied voice (the facilitator's voice is em- } \\
\text { bodied in a dynamic virtual object in the VE, e.g. } \\
\text { through a walkie-talkie or a loudspeaker) }\end{array}$ \\
$\begin{array}{l}\text { - Avatar embodied voice (the facilitator exists in the VE } \\
\text { as a dynamic avatar or person on a video call, towards } \\
\text { which the immersed user feels a sense of social pres- } \\
\text { ence) }\end{array}$ \\
$\begin{array}{l}\text { Spatial communication } \\
\text { she facilitator provides } \\
\text { user's attention to objects } \\
\text { within the virtual environ- } \\
\text { ment. }\end{array}$ & $\begin{array}{l}\text { Non-integrated highlighting (highlighting objects in a } \\
\text { manner that is not at all context-appropriate, e.g. a big } \\
\text { floating arrow or green ring around a virtual object, as } \\
\text { commonly seen in video games) } \\
\text { Integrated highlighting (guiding the user's attention in } \\
\text { a manner that is a natural part of the VE; e.g. a virtual } \\
\text { character appearing and pointing towards the object in } \\
\text { question); }\end{array}$ \\
\hline
\end{tabular}

\section{Post-task questionnaire and interview (15min)}


After completing all of the tasks and escaping from the escape room, the VR users are shown end credits and are then asked to remove the VR headset. They are invited to fill out a post-task presence questionnaire, after which the experiment facilitator will interview them about the escape room experience in general. We are hoping for the participants to point out interruptions or positive incidents during their collaboration tasks which, with linkage to the in-VR presence measurements, can hopefully validate correlation between pleasant immersive VR experience and high level of presence.

\section{Conclusions}

In this paper we have outlined the challenges of conducting collaborative problemsolving in an asymmetric virtual environment. We have identified the key elements promoting and hindering the perceived sense of presence in VR. We argue that high sense of presence is a critical ingredient for successful co-design and collaboration. We have described a VR environment and an experiment that will enable us to test different in-VR presence measurement methods for several identified communication and interaction practices in order to study their impact on the immersed user's sense of presence.

Our future work includes developing the described VR environment, preparing a collection of presence measuring instruments and conducting an extended qualitative user experiment based on the constant comparative method described in the grounded theory [48] that enables us to define, model and validate the most suitable methods for collaboration in asymmetric VR environments.

\section{Acknowledgements}

We wish to thank the department of Computer Science at Aalto University School of Science for funding this research.

\section{References}

1. Heeter C (1992) Being There: The Subjective Experience of Presence. Presence: Teleoperators and Virtual Environments 1:262. https://doi.org/10.1162/pres.1992.1.2.262

2. Lombard M, Ditton T (1997) At the Heart of It All: The Concept of Presence. J Comput Mediat Commun 3:. https://doi.org/10.1111/j.10836101.1997.tb00072.x

3. Slater M (2003) A note on presence terminology

4. Slater M, Khanna P, Mortensen J, Yu I (2009) Visual Realism Enhances Realistic Response in an Immersive Virtual Environment. IEEE Comput Grap Appl 29:76-84. https://doi.org/10.1109/MCG.2009.55

5. Diemer J, Alpers GW, Peperkorn HM, et al (2015) The impact of perception and presence on emotional reactions: a review of research in virtual reality. Front Psychol 6:. https://doi.org/10.3389/fpsyg.2015.00026 
6. Bowman DA, McMahan RP (2007) Virtual Reality: How Much Immersion Is Enough? Computer 40:36-43. https://doi.org/10.1109/MC.2007.257

7. Cummings JJ, Bailenson JN (2016) How Immersive Is Enough? A Meta-Analysis of the Effect of Immersive Technology on User Presence. Media Psychology 19:272-309. https://doi.org/10.1080/15213269.2015.1015740

8. Slater M, Steed A (2000) A Virtual Presence Counter. Presence 9:413-434. https://doi.org/10.1162/105474600566925

9. Zenner A, Speicher M, Klingner S, et al (2018) Immersive Notification Framework: Adaptive \& Plausible Notifications in Virtual Reality. In: Extended Abstracts of the 2018 CHI Conference on Human Factors in Computing Systems. Association for Computing Machinery, Montreal QC, Canada, pp 1-6

10. Milgram P, Kishino F (1994) A taxonomy of mixed reality visual displays. IEICE TRANSACTIONS on Information and Systems 77:1321-1329

11. Cline E (2011) Ready player one, 1st ed. Crown Publishers, New York

12. Gugenheimer J, Stemasov E, Frommel J, Rukzio E (2017) ShareVR: Enabling Co-Located Experiences for Virtual Reality between HMD and Non-HMD Users. In: Proceedings of the 2017 CHI Conference on Human Factors in Computing Systems - CHI '17. ACM Press, Denver, Colorado, USA, pp 4021-4033

13. Lee J, Kim M, Jinmo K (2019) RoleVR: Multi-experience in immersive virtual reality between co-located HMD and non-HMD users. Multimedia Tools and Applications. https://doi.org/10.1007/s11042-019-08220-w

14. Peter M, Horst R, Dörner R (2018) VR-Guide: A Specific User Role for Asymmetric Virtual Reality Setups in Distributed Virtual Reality Applications

15. Slater M, Lotto B, Arnold MM, Sánchez-Vives MV (2009) How we experience immersive virtual environments: the concept of presence and its measurement. Anuario de Psicología, 2009, vol 40, p 193-210

16. Brade J, Lorenz M, Busch M, et al (2017) Being there again - Presence in real and virtual environments and its relation to usability and user experience using a mobile navigation task. International Journal of Human-Computer Studies 101:76-87. https://doi.org/10.1016/j.ijhcs.2017.01.004

17. Rebelo F, Noriega P, Duarte E, Soares M (2012) Using Virtual Reality to Assess User Human factors 54:964-82. https://doi.org/10.1177/0018720812465006

18. Tiainen T, Jouppila T (2019) Use of Virtual Environment and Virtual Prototypes in Co-Design: The Case of Hospital Design. Computers 8:44. https://doi.org/10.3390/computers 8020044

19. Mobach MP (2008) Do virtual worlds create better real worlds? Virtual Reality 12:163-179. https://doi.org/10.1007/s10055-008-0081-2

20. Koller M, Schäfer P, Lochner D, Meixner G (2019) Rich Interactions in Virtual Reality Exposure Therapy: A Pilot-Study evaluating a System for Presentation Training. In: 2019 IEEE International Conference on Healthcare Informatics (ICHI). pp 1-11

21. Ling Y, Nefs HT, Morina N, et al (2014) A meta-analysis on the relationship between self-reported presence and anxiety in virtual reality exposure therapy for 
anxiety disorders. PLoS ONE 9:e96144. https://doi.org/10.1371/journal.pone. 0096144

22. Price $\mathrm{M}$, Anderson $\mathrm{P}$ (2007) The role of presence in virtual reality exposure therapy. J Anxiety 21:742-751. https://doi.org/10.1016/j.janxdis.2006.11.002

23. Botella (2017) Recent Progress in Virtual Reality Exposure Therapy for Phobias: A Systematic Review. - PubMed - NCBI. https://www.ncbi.nlm.nih.gov/pub$\mathrm{med} / 28540594$. Accessed $21 \mathrm{Feb} 2020$

24. Yee N, Bailenson J (2007) The Proteus Effect: The Effect of Transformed SelfRepresentation on Behavior. Human Comm Res 33:271-290. https://doi.org/10.1111/j.1468-2958.2007.00299.x

25. Peck TC, Seinfeld S, Aglioti SM, Slater M (2013) Putting yourself in the skin of a black avatar reduces implicit racial bias. Conscious Cogn 22:779-787. https://doi.org/10.1016/j.concog.2013.04.016

26. Slater M, Antley A, Davison A, et al (2006) A Virtual Reprise of the Stanley Milgram Obedience Experiments. PLoS ONE 1:e39. https://doi.org/10.1371/journal.pone.0000039

27. Slater M (2009) Place illusion and plausibility can lead to realistic behaviour in immersive virtual environments. Philos Trans R Soc Lond B Biol Sci 364:3549_ 3557. https://doi.org/10.1098/rstb.2009.0138

28. Schuemie MJ, van der Straaten P, Krijn M, van der Mast CA (2001) Research on presence in virtual reality: a survey. Cyberpsychol Behav 4:183-201. https://doi.org/10.1089/109493101300117884

29. Samana R, Wallach H, Safir M (2009) The Impact of Personality Traits on the Experience of Presence. pp 1-7

30. Lessiter J, Freeman J, Keogh E, Davidoff J (2001) A Cross-Media Presence Questionnaire: The ITC-Sense of Presence Inventory. Presence 10:282-297. https://doi.org/10.1162/105474601300343612

31. Liszio S, Masuch M (2016) Designing Shared Virtual Reality Gaming Experiences in Local Multi-platform Games. In: ICEC

32. Riches S, Elghany S, Garety P, et al (2019) Factors Affecting Sense of Presence in a Virtual Reality Social Environment: A Qualitative Study. Cyberpsychology, Behavior, and Social Networking 22:288-292. https://doi.org/10.1089/cyber.2018.0128

33. Weech S, Kenny S, Barnett-Cowan M (2019) Presence and Cybersickness in Virtual Reality Are Negatively Related: A Review. Front Psychol 10:. https://doi.org/10.3389/fpsyg.2019.00158

34. IJsselsteijn WA, De Ridder H, Freeman J, Avons SE (2000) Presence: concept, determinants, and measurement. In: Human vision and electronic imaging V. International Society for Optics and Photonics, pp 520-529

35. IJsselsteijn WA (2004) Presence in depth. Technische Universiteit Eindhoven

36. van Baren J, IJsselsteijn W (2004) Measuring Presence: A Guide to Current Measurement Approaches

37. Dillon C, Keogh E, Freeman J, Davidoff J (2001) Presence: Is your heart in it. 4th Int Wkshp on Presence 
38. Lombard M, Bolmarcich T, Weinstein L (2009) Measuring Presence: The Temple Presence Inventory

39. Slater M (2004) How Colorful Was Your Day? Why Questionnaires Cannot Assess Presence in Virtual Environments. Presence 13:484-493. https://doi.org/10.1162/1054746041944849

40. Usoh M, Catena E, Arman S, Slater M (2000) Using Presence Questionnaires in Reality. Presence: Teleoperators and Virtual Environments 9:. https://doi.org/10.1162/105474600566989

41. Schwind V, Knierim P, Tasci C, et al (2017) "These are not my hands!": Effect of Gender on the Perception of Avatar Hands in Virtual Reality. pp 1577-1582

42. Schwind V, Knierim P, Haas N, Henze N (2019) Using Presence Questionnaires in Virtual Reality

43. Schwind V, Knierim P, Chuang L, Henze N (2017) "Where's Pinky?": The Effects of a Reduced Number of Fingers in Virtual Reality. pp 507-515

44. Dahlbäck N, Jönsson A, Ahrenberg L (1993) Wizard of Oz studies: why and how. In: Proceedings of the 1st international conference on Intelligent user interfaces - IUI '93. ACM Press, Orlando, Florida, United States, pp 193-200

45. Dow S, Lee J, Oezbek C, et al (2005) Wizard of Oz interfaces for mixed reality applications. In: CHI '05 Extended Abstracts on Human Factors in Computing Systems. Association for Computing Machinery, Portland, OR, USA, pp 13391342

46. Klemmer SR, Sinha AK, Chen J, et al (2000) Suede: a Wizard of Oz prototyping tool for speech user interfaces. In: Proceedings of the 13th annual ACM symposium on User interface software and technology. Association for Computing Machinery, San Diego, California, USA, pp 1-10

47. Nielsen J (1993) Usability Engineering. Morgan Kaufmann Publishers Inc., San Francisco, CA, USA

48. Glaser BG, Strauss AL (1967) The discovery of grounded theory: Strategies for qualitative research. Aldine de Gruyter, London, UK 